\title{
Postural Optimization for an Ergonomic Human-Robot Interaction
}

\author{
Baptiste Busch $^{1}$, Guilherme Maeda ${ }^{2}$, Yoan Mollard ${ }^{1}$, Marie Demangeat ${ }^{1}$ and Manuel Lopes ${ }^{3}$
}

\begin{abstract}
In human-robot collaboration the robot's behavior impacts the worker's safety, comfort and acceptance of the robotic system. In this paper we address the problem of how to improve the worker's posture during human-robot collaboration. Using postural assessment techniques, and a personalized human kinematic model, we optimize the model body posture to fulfill a task while avoiding uncomfortable or unsafe postures. We then derive a robotic behavior that leads the worker towards that improved posture. We validate our approach in an experiment involving a joint task with 39 human subjects and a Baxter torso-humanoid robot.
\end{abstract}

\section{INTRODUCTION}

Humans and robots are starting to work in shared environments allowing an increasing diversity of tasks they can accomplish together. This also creates new challenges of safety, and acceptability. New robotic assistants should be able to prevent worker's discomfort as well as more aggravated work-related diseases. Some efforts have already been made to improve acceptability by including social behaviors, like proxemics, in the field of human-robot interaction [1]-[3]. Most advanced robotic planners combine multiple notions, including safety and social acceptance, to create humanaware robotic responses [4]. In contrast to a human-human interaction, where both parties try to maximize their own comfort, sometimes at the expense of the other, an assistive robot should always maximize the comfort of its coworker.

A simple example arises when the robot passes, or presents, an object to the human. When handing over an object, the final object pose influences the way we can grasp it [5], [6], and thus the comfort of that posture. One way to solve this problem is to analyze how the human receiver grasps a specific object and orient it to improve the receiving posture [7]. Another way is to define a cost function that captures all possible hand-over configurations and allows to find the optimal comfortable solution. Common cost functions comprise safety, visibility, and postural comfort [4] or naturalness \& appropriateness [8]. However, such techniques rely on a generic human model which might not capture the individual characteristics of each user. We believe that having a personalized model to adapt to the specificity of each user would greatly benefit the interaction. Recent research addressed the problem of a personalized human model to reason on human kinematics while planning the

\footnotetext{
${ }^{1}$ Baptiste Busch, Yoan Mollard, and Marie Demangeat are with FLOWERS Team, Inria, Talence, France firstname. lastnamedinria.fr

${ }^{2}$ Guilherme Maeda is with Technical University of Darmstadt, Germany, maedalias.tu-darmstadt.de

${ }^{3}$ Manuel Lopes is with INESC-ID, Instituto Superior Técnico, Universidade de Lisboa, Portugal manuel. lopes@tecnico.ulisboa.pt
}

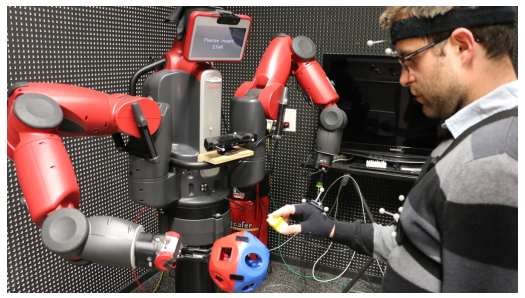

Fig. 1: Representation of the setup considered in the user study. The robot presents to the user a spherical toy in which multiple shapes can be inserted. Final pose of the object is calculated from the user posture at his current location.

robot's behavior [9]. On the other hand, the cost function considered by the authors was chosen for its simplicity and did not include social or postural cost. As they stated, using a more biologically-plausible cost would yield strictly superior results.

"Musculoskeletal disorders" (MSDs) are the single largest category of work-related diseases in many industrial countries [10]. Over the years, many assessment techniques have been developed to evaluate worker's body posture at their workstation [11]. Methods for postural assessment can be classified according to the accuracy of data collection and the degree of intrusion caused by the required sensors to collect them [12], [13]. Observational methods, based on observations of the worker, are straightforward to use and applicable to a large variety of situations [14], [15]. Their accuracy is however limited as they are mainly developed for paper-based observations without the need of specific tools or sensors. With the development of non-intrusive human tracking system, like depth sensors, automatic postural assessment based on observational methods start to emerge [16]. The development of such techniques will have a significant impact in industry by drastically reducing the cost of ergonomic solutions. Moreover, it will also benefit fields like human-robot interaction by allowing a more thorough analysis of the human coworker.

When we, humans, accomplish a task our body posture is (partially) constrained. For example, acting on an object constrains the pose of the hand relatively to the object and the head faces the object we are acting upon [17]. But due to the large number of degrees of freedom (DOF) of the human body, other body parts are unconstrained and several body postures are viable with respect to the task. However, not all of them are viable in terms of ergonomics. Optimizing the model body posture is then the logical next step to find an ergonomically correct posture for the worker to accomplish 
a specific task.

Most researches on human-robot interaction optimize the robot's placement and behaviors w.r.t specific cost functions that acknowledge human needs [4], [18], [19]. Similarly, our idea is to find an optimal human body posture to solve a task considering three important aspects of the interaction that are safety, acceptability and task constraints. Those three factors were chosen according to their importance in the existing literature and are very similar to previous researches in the human-robot interaction field [8], [20]. Our hypotheses, verified in this work, is that the user will intuitively behave similarly to the predictions made by our algorithm. In the case of handing over objects or joint manipulation, the robot can induce the human location and posture by planning the positioning of the object within the shared workspace.

In this paper we introduce a framework for postural optimization of the human body. This framework respects the three aspects of the interaction by introducing notions derived from proxemics [1], subject laterality, and visibility [17] for the notion of acceptability. Other measures such as legibility could also be considered, as we did in our previous work [21], but here we focus more on physical comfort and not on the impact of perception of intentions in the shared activity. Safety, only considered here as the postural risk of MSDs, is handled by the Rapid Entire Body Assessment (REBA) technique [15]. The choice of this postural assessment technique was motivated by its range of application and the fact that it provides quantitative measures.

This work aims at being a study on the effects of incorporating postural assessment techniques derived from ergonomic researches in the human-robot interaction. The main contribution of our work is the integration of the REBA method in a planning framework to provide a realistic estimate of a motion's ergonomic cost. Second, we introduce a solution to automatically assess the worker's body posture using the same REBA method and couple it to a graphical interface to feedback the user on the risk of his or her posture. This graphical interface is presented in Section IV. Finally we have realized an extensive user study with 39 participants that validate the benefits of our approach on the interaction. We have collected both the subject's perception of the system and quantitative measures provided by the automatic assessment of the subject's posture and analyzed them in Section VI.

Closer to our work is the research of Sisbot et al. [4], [19], [20] and the recent works from Bestick et al. [9] and Suay et al. [22]. With the exception of [22], the cost function we have chosen to respresent the human factor is more biologically grounded and taken directly from the ergonomic field. Moreover, our implementation of the visibility cost, firstly introduced by Sisbot et al., also optimizes the object orientation and not only its position. This is particularly important for the object used in our user study, illustrated in Fig. 1]

Details of the implementation are given in Section $\mathrm{V}$ The framework has been tested on a user study, introduced in
Section VI by comparing different methods for handing over the spherical object illustrated in Fig. 1.

\section{Automatic Postural Assessment}

Automatic postural assessment, based on observational methods, has started growing in interest with the development of cheap and easy to use human tracking systems [23]. Although the REBA technique has been initially developed for pen-paper observations [15], the fact that it handles static as well as dynamic postures and that it relies on quantitative values makes it suitable for an automatic assessment. The technique is based on the observation of joints of the human body. Each joint value is associated with a score which corresponds to a risk of MSDs. The higher the score, the higher the risk. As a high score on the upper arm is not equivalent to the same score on the trunk, the method also provides comparative tables to calculate the final score that ranges from 1 (no risk of MSDs) to 12 (very high risk, recommendation to change posture immediately).

To be fully automatized, the technique requires a human kinematic model that returns the joint values from the specific part of the human body (e.g. elbows, hands...). In Section $\mathrm{V}$ we detail the process of creating a personalized human model based on Kinect skeleton model. Joint values and REBA score of the observed posture are calculated from the tracked frames.

This assessment technique is the main component of our framework for postural optimization detailed in Section III. While other techniques for MSD assessment are available, here we opt to use the REBA metric due to its generality and the provision of a quantitative assessment.

\section{Postural Optimization}

To respect the three main aspects of interaction, that are safety, acceptability, and task constraints, we define several cost functions and constraints to find an optimal body posture during the interaction. In the following paragraphs we detail, for each aspect, the associated cost function or constraint.

\section{A. Safety and Comfort}

Human safety is a central piece of the interaction. In term of body posture, safety corresponds to minimizing the risk of MSDs. To achieve this we find the body posture that minimizes the score returned by the postural assessment technique REBA [15].

In its original formulation, the REBA method does not show suited for numerical optimization due to the discrete grading and the presence of plateaus. Thus, we approximate the discontinuous nature of the scores by a sum of weighted polynomials, and optimize the weights to closely reproduce the score in the continuous domain,

$$
C_{\text {posture }}(q)=\sum_{i=1}^{n} w_{i} Q_{i}(q)
$$

Where $n$ is the number of joints considered in the REBA techniques and $Q_{i}(q)$ is a 2 nd order polynomial of joint $i$. Coefficients of the polynomials are calculated from the 
original REBA score of each joint. The weights $w_{i}$ are learned from the total REBA score calculated on several body configurations. For learning the weights, we define a set of random poses, ensuring each class of the original REBA cost (from 1 to 12 ) are evenly represented. We then find the weights that minimize the difference between the original REBA cost and its polynomial approximation for all the posture of the set.

The posture of the body, represented by its vector of joints $q$ is considered as being static and independent from the initial body configuration. We only consider static postures. This choice was motivated by the fact that any initial body configuration might be ergonomically incorrect and therefore should not interfere with the final posture.

Although the cost function (1) is an approximation of the REBA score, postures that minimize it also minimizes the original score as the minima of the two functions are identical. In the rest of this paper we use the score function (1) to optimize the body posture but assess the obtained posture with the original REBA method.

In its implementation the REBA method also takes the weight of the load into consideration. For the moment, we have discarded it in our experiments as it was considered as negligible. However, this is an important notion to add in the future.

\section{B. Task constraints}

For some tasks, the pose of a specific body part, like the hand, is constrained to a specific location. For example when we use a tool our hand is tied to it, which itself is linked to the object we are acting upon. This part of the framework is the only specific part that needs to be specified for each task. Most common task constraints would be to set parts of the worker's body to a certain pose.

Considering the forward kinematic of our human model $F K$, the pose in Cartesian space of body part $i$ is represented as $F K_{i}$. Constraining a body part to a certain location in space is equivalent to minimizing the distance between the forward kinematic and the desired pose $F K_{i}^{d e s}$. This distance is expressed as two subcomponents, one for the position as Euclidean distance and one for the orientation as distance between two quaternions. The distance between two quaternions is defined as $\left(Q_{1}, Q_{2}\right)=1-\left\langle Q_{1}, Q_{2}\right\rangle^{2}$. Constraining multiple body parts is performed by summation of the distances,

$$
\begin{aligned}
C_{\text {task }}\left(F K, F K^{\text {des }}\right)= & \sum_{i}\left(\left\|F K_{i, \text { pos }}-F K_{i, \text { pos }}^{\text {des }}\right\|\right. \\
& \left.+k\left(1-\left\langle F K_{i, \text { rot }}, F K_{i, r o t}^{\text {des }}\right\rangle^{2}\right)\right) .
\end{aligned}
$$

Because position and orientation distances do not share the same unit, it requires the addition of a scaling factor $k \in \mathbb{R}$. In practice, we have set $k \approx \pi$. As the quaternion distance varies between 0 and 1 , this then corresponds to the distance between the two angles traveled on the unit circle. We also note that $F K^{\text {des }}$ might be expressed w.r.t the object frame. This is particularly interesting when the object is free to move in space and its pose is part of the optimization.

\section{Acceptability}

The concept of acceptability is not easy to define. It is usually divided into social acceptability: How does the society perceive robotic systems?, and practical acceptability: How do people perceive the robot when interacting with it? [24]. In this paper we focus on the latter. We incorporate visibility, proxemics and user's laterality (right or left-handed) in our framework in order for the worker to feel at ease during the interaction.

Visibility: Visibility is an important notion to consider. When the robot moves an object, it should always do so in the worker's field of view. Otherwise, it might surprise the user. We have put the visibility notion under acceptability although it does not uniquely belong to this category. For example, a non trained worker would most probably look at the object he is acting upon in order to fulfill his task (task constraints). Such as novices in computers look at their keyboard when typing. Another one might get hurt if he uses some tools and cannot visually verify the results of his actions (safety).

To optimize for visibility, the model's head pose is directly linked to the pose of the object $X_{o b j}$ acted upon. Pose of the object can be considered as fixed, attached to the model's hand by a predefined transformation or set as a variable of the optimization. We denote the sight vector coming from the head as $\vec{H}_{x}, H$ corresponding to the forward kinematic of the head $F K_{\text {head }}$. We consider that this vector originates from the center of the head and is parallel to the ground when the head is straight. The visibility cost corresponds to the angle between the vector from the center of the head to the object $X_{o b j} H$ and $\vec{H}_{x}$. It is calculated using the dot product between them as $\arccos \left(\vec{H}_{x} \cdot X_{o b j} H\right)$. This way we ensure that the object is approximately at the center of the vision cone when this cost is close to 0 .

For some objects, the orientation also matters. For example, the handle of a tool should be directed toward the user to ease the grasping. To represent this we consider a feature vector $\vec{v}_{o b j}$ that need to be aligned with the directional vector of the head $\vec{H}_{x}$. At the moment, this vector is predefined for each object used in the experiments. The corresponding cost is also defined by the dot product $\arccos \left(\vec{H}_{x} \cdot \vec{v}_{o b j}\right)$. The final cost for visibility corresponds to the sum of the two dot products,

$$
\begin{aligned}
C_{v i s i b i l i t y}\left(F K_{H e a d}, X_{o b j}\right)= & \arccos \left(\vec{H}_{x} \cdot X_{o b j} H\right) \\
& +\arccos \left(\vec{H}_{x} \cdot v_{o b j}\right) .
\end{aligned}
$$

Proxemics: People have special concerns about their personal space either in terms of intimacy and safety. We consider proxemics - physical and psychological distancing from others [25] - to address such concerns. Close interaction like handing-over an object would be socially unacceptable and create uncomfortable situations if it happens too close to the worker, especially for non-trained ones. 
In our framework we have added the proxemics notion as a constraint to ensure that the optimized posture will not violate the worker's intimate space. The proxemics constraint corresponds to the minimal distance from the human waist to the object. Only planar distance ( $\mathrm{x}$ and y coordinates) are here constrained. This limit correspond to the boundary of the subject's intimate space, i.e $0.45 \mathrm{~m}$ [26],

$$
X_{O b j} \in \text { proxemics_constraints. }
$$

Laterality: Every worker is unique. Some are righthanded, other left-handed. Therefore, the model should also take into account this specificity. We leave the possibility to select the worker's laterality. This impacts which hand is constrained to act upon an object.

\section{Framework definition}

Finally, we group all the previous cost functions and constraints (1,3) to define a parameterized framework, whose pseudocode is detailed in algorithm 11, for optimizing the human body posture while solving a task. Our formalism is very generic and considers many tasks beyond the ones presented in this paper. The task constraint function and its parameters are the parts that have to be redefined depending on the specific task to solve.

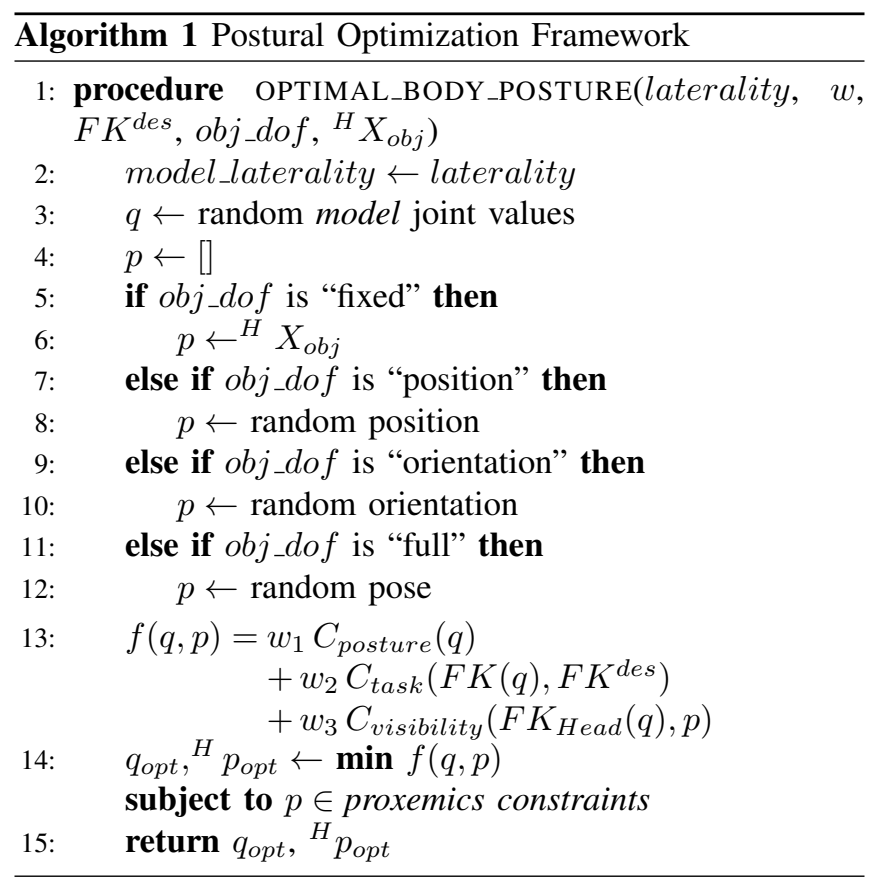

The final cost function is a weighted sum of the different elements, some of them with different units for which we need different weights $w_{i} \in \mathbb{R}$. This weights will most probably be task dependent. For example, in some industrial tasks, visibility might be a critical aspect. In this case increasing the weight that account for visibility will lead to the desired behavior. The outputs of the algorithm $q_{o p t}$ and ${ }^{H} p_{\text {opt }}$ correspond to the optimal human posture in joint space and the optimal object pose w.r.t the human reference frame respectively.

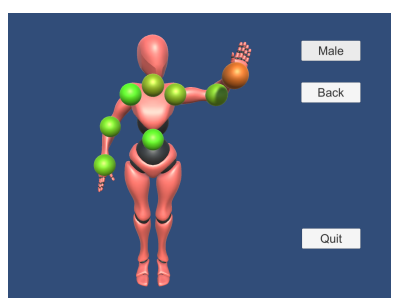

female model, front view

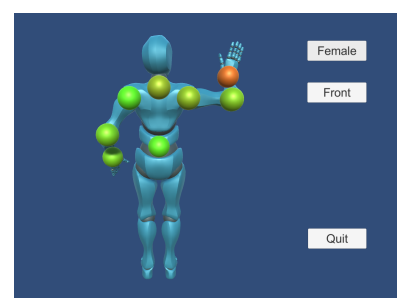

male model, back view
Fig. 2: Screenshots of the graphical interface in two possible configurations. The same body posture is used in both pictures. In front view, by contrast with the back view, the body configuration mirrors the real posture.

\section{FEEDBACK INTERFACE}

Most of the time, workers are unaware of the risk induced by a wrong posture. Dangerous postures might not have a direct effect on the body but it is the repetition that increases the risk of MSDs. Therefore, a direct feedback is an interesting feature that could help workers maintaining a good posture at their workstation. To that extent, we have developed a graphical interface to animate a model with the user's observed body posture. The projection on the model is almost real-time. We also evaluate the risk of his or her posture, using the REBA assessment technique, and displays it with spheres located at the evaluated body joints. The color of each sphere represents the risk at its corresponding location. It ranges from "green" (no risk) to "red" (high risk) according to the REBA method. In Fig. 2 we show two screenshots of the graphical interface. Using the buttons on the right side, the user can choose to display a male or a female version of the model. He or she can also switch between a front or a back view. After some trials with test subjects, it has been decided that the front view should mirror the user's body configuration, i.e rising the right arm translates to a rising of the model left arm. In back view, however, the body configuration is normally displayed.

\section{IMPLEMENTATION DETAILS}

In this section we define the implementation choices we have made to track the human body for the automatic assessment of Section $\mathrm{II}$ and to optimize the body posture using the framework defined in Section III.

\section{A. Model generation and human tracking}

The automatic postural assessment requires a human model that returns joint values from pose of body segments. To this end, we calibrate a personalized human model as a URDF with 32 degrees of freedom (DOF) by calculating the length of each body segments on the observed subjects skeleton using Kinect cameras. This personalized model is then used during the optimization described in Section III. While during the calibration of the model the environment can be setup such that the human can stand directly in front the camera, during the execution, we found that the presence of the robot, constant occlusions, and space constraints make 
it difficult to reliably assess the REBA score using the same camera.

Thus, currently, at runtime we opted to track the human using optical markers ${ }^{1}$ Each subject has been equipped with a suit covered by OptiTrack markers to track the main body segments (head, hands, elbows, torso and waist). The models frames are recorded during the whole interaction. To obtain the model joint values we compute its inverse kinematic on the recorded data. As the legs are not tracked, we set them in rest position, i.e. with joint values leading to the minimal REBA score.

The waist frame is the most important frame of the OptiTrack suit. It corresponds to the model base frame. We also use it to express the position and orientation of the subject in the robot workspace.

\section{B. Optimization of the cost functions}

To minimize the costs defined in Algorithm 1 we use a simple gradient descent algorithm from scipy library. This is sufficient as we consider only static postures and not trajectories.

\section{From human to robot space}

The solution provided by Algorithm 1 corresponds to the optimal body posture in joint space, $q_{o p t}$ and the optimal object pose ${ }^{H} p_{\text {opt }}$ in the human space. We must now generate a robot trajectory that positions and orients the object in its optimal location ${ }^{H} p_{o p t}$ such that the human is in the optimal posture $q_{o p t}$. The relation between $q_{o p t}$ and ${ }^{H} p_{\text {opt }}$ is defined as the forward kinematic $F K$. The robot trajectory can be designed by expressing the pose of the object w.r.t the robot's reference frame, ${ }^{R} p_{\text {opt }}$. To this end, at runtime we locate the current position of the human w.r.t the robot's frame using, for example, optical markers or depth cameras to compute a transformation ${ }^{R} T_{H}$. This process is illustrated in the left part of Fig. 3 .

Transformed into the robot's space, the optimized solution represents the optimal posture for the human to interact with the robot. For simplicity, take the case where ${ }^{R} p_{\text {opt }}$ needs to be satisfied only at the end of the interaction (e.g. in handover) and the robot starts from a pre-defined home position ${ }^{R} p_{\text {init }}$. Depending on the location of the human w.r.t. the robot, and the degree of redundancy of the robot, two situations are possible. If the final object position is within the workspace of the robot, many possible robot trajectories that lead to the satisfaction of ${ }^{R} p_{\text {opt }}$ can be designed. Conversely, if the final object position is outside the workspace of the robot, no trajectory can be generated.

To account for both cases and quickly generate a trajectory, we solve a motion planning problem where the initial and final end-effector positions of the robot are connected by a straight line (in Cartesian space), as an initial guess. The orientation of the end-effector is interpolated by slerp between the initial and final poses. If an IK solution on this straight

\footnotetext{
${ }^{1}$ We are working on replacing the optical markers by Kinect cameras also during the execution, creating a less invasive measurement system that can be seamlessly integrated in real industrial scenarios, for example.
}

Mapping to robot's reference frame
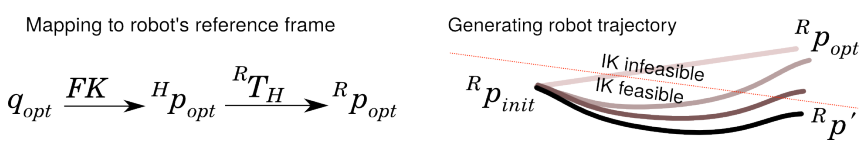

Fig. 3: Left: finding the desired object pose in Cartesian space w.r.t. the robot's reference frame ${ }^{R} p_{\text {opt }}$ given the solution of the ergonomic optimization $q_{o p t}$ and ${ }^{H} p_{\text {opt }}$. Right: a straight trajectory is used as an initial guess to bring the object from the robot's home position to the handover position. If a solution is not possible, stochastic optimization is used to optimize the trajectory.

trajectory is not found, we use stochastic optimization to slightly perturb the final desired position of the end-effector and also to reshape the straight line to search for a valid robot's IK trajectory 2 Perturbing the final desired position of the end-effector will make the exact reproduction of ${ }^{R} p_{\text {opt }}$ and force the human model to deviate from its optimal posture $q_{\text {opt }}$. Thus, we penalize deviations from the original desired location to find a compromise between the optimal posture of the human and a kinematically feasible robot trajectory. This process is illustrated in the right part of Fig. 3.

\section{Graphical interface}

The feedback graphical introduced in Section IV] has been developed using Unity software. The male and female model were imported from Mixamo website without license nor restriction of use. The code for the graphical interface is available on a GitHub repository 3 .

\section{USER-STUDY EXPERIMENT}

We apply the postural optimization framework on an experiment with real users interacting with our Baxter torsohumanoid robot. Two points are tested: Are the postures generated with our framework perceived better than other types of postures? and Does the optimal robot's behavior leads the real user to a safer posture? During those experiments, the feedback interface was not presented to the subjects to avoid perceptual biases toward the postures generated by our algorithm.

With the object illustrated in Fig. 1] we mimic an industrial scenario where the robot helps the worker by positioning and orienting an object in which the worker has to insert specific shapes. In total five shapes were considered, located on the blue side of the ball. Name of the shape to insert was written on the robot's screen. Between each insertion the robot was sent back to a neutral pose to let the user prepare the next shape.

We have conducted an extensive user study with 39 participants (18 females and 21 males, 2 left-handed, aged $35 \pm 11.26$ ). Before the experiment, subjects were asked to rate their experience with robotic systems on a scale from 1 (no experience) to 5 (daily usage). Result is $2.18 \pm 1.27$.

\footnotetext{
${ }^{2}$ details of this method can be found in [27]

${ }^{3}$ https://github.com/3rdHand-project/PosturalFeedbackInterface
} 


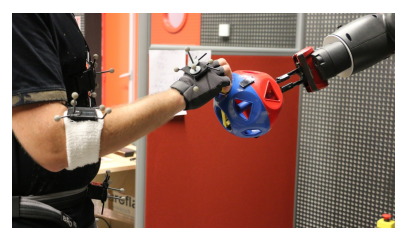

Relative pose

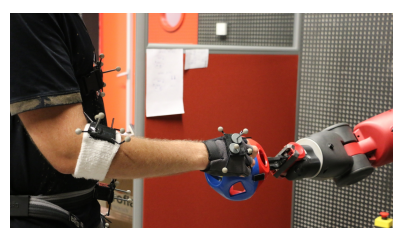

Optimized pose
Fig. 4: Difference between the pose obtained with the relative (left picture) and the optimized conditions (right picture).

Three robot's behaviors have been compared. We refer to them as fixed, relative and optimized conditions. Each participant has been presented a sequence of two behaviors from the set of all permutations. Each run of a behavior is composed by the five shapes to insert in a random sequence. Behaviors are detailed in the following paragraphs:

Fixed: In this behavior the robot does not take into account the worker position and deliver the object to a fixed pose. We have used this behavior as a baseline.

Relative: Research from ergonomics tells us that handing-over an object should happen at torso height at twothird of the receiver's arm maximum elongation [6], [28]. For orientation, we constrained the insertion hole to face the user. At execution, the user's torso frame is tracked and the pose of the object on the reference frame of the robot is computed using the required homogeneous transformations.

Optimized: Using our framework from Sec. III we optimize the user's body posture to receive the object and calculate where to deliver it with reference frame transformations. Computing the optimization of the receiving pose is achieved within $\sim 5 s$ on a $7-3720 \mathrm{QM} 2.60 \mathrm{GHz}$ computer.

After observing the two behaviors, the subjects were asked to order them according to their preferences and to complete a survey composed of a 12 Likert scale items for each observed behavior. Affirmations were ranked from 1 (I strongly disagree) to 5 (I totally agree). They are based on the System Usability Scale methodology [29] with one affirmation over two written with the negative form. The survey was divided in three subcategories representing the considered aspect of the interaction, i.e safety, acceptability and task constraints. This separation was not made visible to the subjects for whom the 12 affirmations were shown in a random order. After answering the 12 items, subjects had the possibility to leave comments on the observed behaviors. Complete results and survey questions are detailed in Fig 5 . For clarity, the affirmations in the negative form are drawn with negative values from -5 to -1 . Subjects' body poses were recorded, using the OptiTrack suit, to evaluate their posture during accomplishment of the task.

The data of the experiment are available on a Zenodo repository $\sqrt{4}$ alongside a GitHub repository that contains all the necessary materials to read them ${ }^{5}$ A video of the

\footnotetext{
${ }^{4}$ https://doi.org/10.5281/zenodo.321599

${ }^{5}$ https://github.com/3rdHand-project/PosturalOptimizationDataReader
}

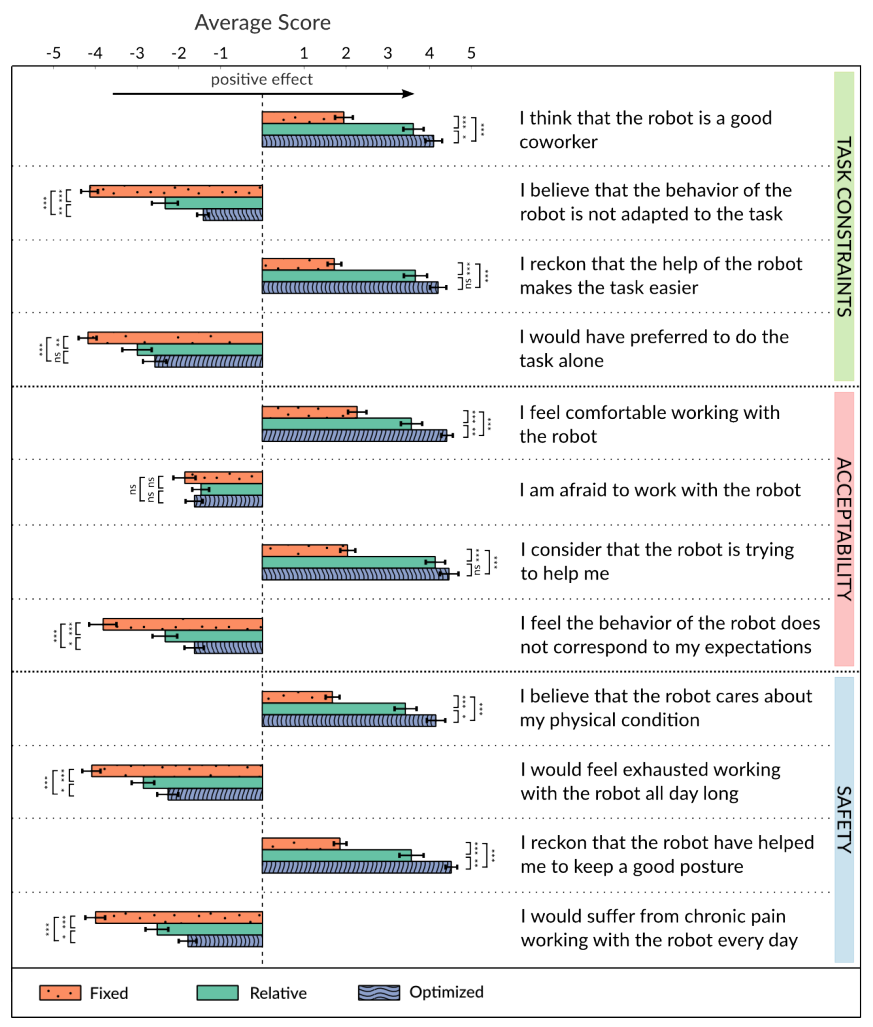

Fig. 5: Average results and standard error of the mean for the responses to the survey. Each subject have observed two of the three behaviors in a random order. Affirmations are Likert scale items, based on the SUS methodology, ranked from 1 to 5 [29]. The negative values for the affirmations in negative form only appear in the figure for clarity matters. Significance have been tested using Mann-Whitney U test and noted according to the standard defined by the APA(American Psychological Association).

experiment is available on our Vimeo channe $]^{6}$

\section{Qualitative Results}

From the survey results Fig 5 and the recorded data we want to validate the three following hypotheses:

- The Fixed condition should have the lower rank for all the considered aspects as it does not respect the task constraints and do not account for user's safety $\left(H_{0}\right)$.

- In terms of task constraints and acceptability the relative and optimized conditions should produce similar results. Main differences between them should arise from safety related affirmations $\left(H_{1}\right)$.

- On the recorded data the optimized condition should have the lowest REBA value $\left(\mathrm{H}_{2}\right)$.

Almost all subjects who have observed the optimized condition have put it first in their preference ordering. Only one person, who got the relative and the optimized conditions, has chosen the relative one first. Second preferred method is the relative condition. The Fixed condition has never been ranked first. For all the affirmations in Fig 5 there is a

\footnotetext{
${ }^{6}$ https://vimeo.com/163699896
} 
tendency for the optimized condition to be preferred over the two other ones. Results have been compared using MannWhitney $U$ test and show a significant preference for the optimized condition when summing up all the affirmations ( $p$-values $<0.05$ for the three combinations). The order of the conditions does not influence the results $(p-$ values $\approx$ 0.5 for the three method). Due to the small number of left-handed people we cannot check for any significant differences between laterality.

Considering the results for the three subcategories, there is no significant differences between the optimized and the relative conditions for the questions on task constraints and acceptability ( $p-$ values $=0.19$ and 0.33 respectively). All the other combinations are significantly different.

Fig. 4 shows the difference between the pose obtained with the relative and the optimized conditions. In the relative condition, we observe that the interaction happens at a higher level and forces the subject to bend his wrist.

\section{Quantitative Results}

During all the process the subjects' body motions have been recorded and their posture evaluated using the REBA method. We only consider the moment of insertion and average over the few time-steps it has taken the subject to do this action. The moment of insertion was automatically detected from the Cartesian distance between the subject's dominant hand (the one that have been used to insert the shape) and the center of the ball. We arbitrarily set that distance to be less than $20 \mathrm{~cm}$ for the time-step to be accepted as an insertion. Fig 6 shows the averaged REBA score over all the subjects for the three behaviors for some parts of the body. Differences between the conditions have been tested using a paired t-test on paired data from subjects, merging conditions in opposite order. For example, statistical differences between the fixed and the optimized conditions have been obtained from people that have observed the fixed then the optimized conditions and people that have observed the optimized then the fixed conditions.

The data in Fig 6 show a significantly lower score on shoulder, wrist and total values for the optimized condition. The Relative condition presents the highest wrist score. In their comments some subjects have written that their wrist was not in a correct pose when they had to insert the shapes. Especially one subject that had a wrist tendinitis said he felt some discomfort.

\section{Analysis}

From the results of the user study we validate the three hypotheses enunciated previously. The fixed condition is the worst possible behavior both in terms of subject's perception and REBA score $\left(H_{0}\right)$. Although relative condition seems to be an acceptable solution it produces a significantly higher body posture score, especially on the wrist. In terms of acceptability and task constraints it is perceived similarly to the optimized solution returned by the framework. Yet in terms of user's safety it performs significantly worst $\left(H_{1}\right)$.

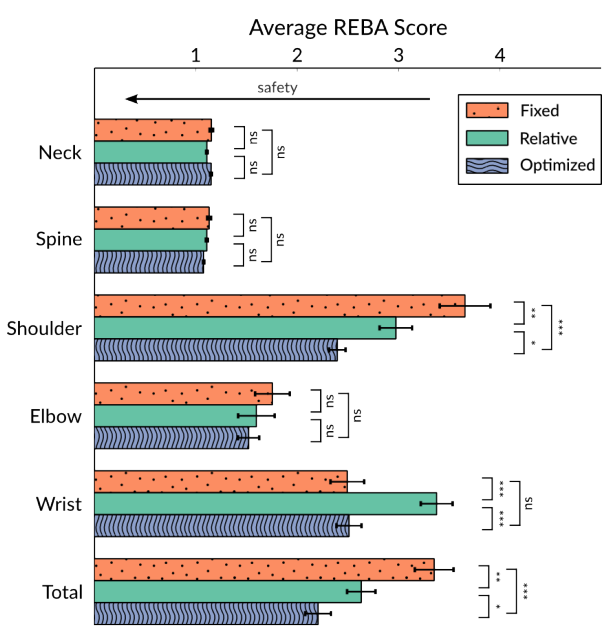

Fig. 6: Average score and standard error of the mean of the recorded body posture using the REBA method. Only dominant arm have been included in the figure for clarity, but data for the opposite arm are also available. Significance have been tested using Wilcoxon test on paired data. The recorded postures for the optimized condition present a lower REBA score. The relative condition leads to an higher wrist score that can create some discomfort.

From the analysis of the recorded data, we observe that the optimized condition significantly reduces the body posture cost. There is 1.1 point of reduction (3.35 to 2.21 in average) compared to the fixed condition and 0.4 point (2.63 to 2.21 ) compared to the relative condition. Although this is not a huge improvement, we have to note that only the dominant arm is used to solve this task. We can expect better results for some task that put a strain on all the body parts. Nevertheless, the repetition of a suboptimal posture can increase the risk of MSDs. Interestingly, the higher wrist score for the relative condition can be linked to the comments made by the subjects. During the insertion their wrist was put in extension due to the orientation of the insertion hole. This have been correctly detected by the assessment technique and it is the main weakness of the relative condition.

Obviously the relative condition results could be improved by changing the orientation that causes the wrist discomfort. But then this would require to empirically find a "correct" solution for each task considered. For some of them this might be not trivial. Therefore, the genericity of our framework allows to automatically find better postures in any task.

\section{CONCLUSION}

For a safe and comfortable human-robot interaction it is important to respect three aspects of the interaction: task constraints, safety and acceptability. The growing number of MSDs shows that people might be unaware of the risk of their working posture. By optimizing the human posture to minimize that risk, we can deduce robotic behaviors that are safer in the long-term.

In our work, we introduce a postural optimization framework that account for task constraints and acceptability 
while minimizing the risk of MSDs. For the latest we rely on a personalized human model and postural assessment techniques developed for ergonomic, mainly the REBA assessment method [15]. Coupled to a motion tracking system we also assess the worker's posture during the interaction. We have validated our approach in a user study with 39 volunteers interacting with a Baxter torso-humanoid robot.

Results of the user study prove the efficiency of our framework. It successfully generates body postures to solve the considered task. Moreover, not only are the obtained posture perceived as better solution compared to naive approaches, the real subject's posture are also rated better with the REBA method. The main strength of the framework is to not provide an a priori knowledge on the object pose in order to solve the task. By only defining the task constraints, the framework provides the optimal object location for interaction. However, some limitations are worth noting. One of the actual limitation arises when the robot cannot reach the desired position. For a mobile robot this problem can probably be solved by moving the robot to another location. For a fixed robot the solution would be to reorganized the working cell. If the worker can move in the workspace, then we should find his optimal placement. Second limitation is that our method only consider static posture, although motions with a high dynamic present a high risk of MSDs. Finally, a current limitation of the method is the use of marker tracking, which may hinder the application in real industrial scenarios. Thus, we are currently investigating alternative non-intrusive methods that work in close range.

The graphical feedback interface presented in the paper also requires an extensive study to validate its usage. To avoid potential biases it was turned off during the conducted user study. Therefore, an extension of the work is to analyze how it impacts the worker's posture during the execution of a task.

\section{ACKNOWLEDGMENTS}

This work was supported by national funds through Fundação para a Ciência e a Tecnologia (FCT) with reference UID/CEC/50021/2013 and by the EU FP7-ICT project 3rdHand under grant agreement no 610878.

\section{REFERENCES}

[1] N. Mitsunaga et al., "Adapting robot behavior for human-robot interaction," IEEE Transactions on Robotics, vol. 24, no. 4, pp. 911-916, 2008.

[2] L. Takayama and C. Pantofaru, "Influences on proxemic behaviours in human-robot interaction," in Proc. IEEE International Conference on Intelligent Robots and Systems(IROS'09), St. Louis, MO, USA, 2009, pp. 494-499.

[3] K. Koay et al., "Social roles and baseline proxemic preferences for a domestic service robot," International Journal of Social Robotics, vol. 6, no. 4, pp. 469-488, 2014.

[4] E. Sisbot et al., "Synthesizing robot motions adapted to human presence: A planning and control framework for safe and socially acceptable robot motions," International Journal of Social Robotics, vol. 2, no. 3, pp. 329-343, 2010.

[5] D. Rosenbaum, C. V. Heugten, and G. Caldwell, "From cognition to biomechanics and back: The end-state comfort effect and the middleis-faster effect," Acta Psychologica, vol. 94, no. 1, pp. 59-85, 1996.
[6] M. Kolsch, A. Beall, and M. Turk, "The postural comfort zone for reaching gestures," in Proc. Human Factors and Ergonomics Society Annual Meeting, vol. 47, no. 4, 2003, pp. 787-791.

[7] J. Aleotti, V. Micelli, and S. Caselli, "Comfortable robot to human object hand-over," in Proc. IEEE International Workshop on Robot and Human Interactive Communication (ROMAN'12), Paris, France, 2012, pp. 771-776.

[8] M. Cakmak et al., "Human preferences for robot-human hand-over configurations," in Proc. IEEE International Conference on Intelligent Robots and Systems (IROS'11), San Francisco, California, USA, 2011, pp. 1986-1993.

[9] A. Bestick et al., "Personalized kinematics for human-robot collaborative manipulation," in Proc. IEEE International Conference on Intelligent Robots and Systems (IROS'15), Hamburg, Germany, 2015, pp. 1037-1044.

[10] L. Punnett and D. Wegman, "Work-related musculoskeletal disorders: The epidemiologic evidence and the debate," Journal of Electromyography and Kinesiology, vol. 14, no. 1, pp. 13-23, 2004.

[11] E. Takala et al., "Systematic evaluation of observational methods assessing biomechanical exposures at work," Scandinavian Journal of Work, Environment and Health, vol. 36, no. 1, pp. 3-24, 2010.

[12] A. V. D. Beek and M. Frings-Dresen, "Assessment of mechanical exposure in ergonomic epidemiology." Occupational and environmental medicine, vol. 55, no. 5, pp. 291-299, 1998.

[13] R. Wells et al., "Assessment of physical work load in epidemiologic studies: Common measurement metrics for exposure assessment," Ergonomics, vol. 40, no. 1, pp. 51-61, 1997.

[14] O. Karhu, P. Kansi, and I. Kuorinka, "Correcting working postures in industry: A practical method for analysis," Applied Ergonomics, vol. 8, no. 4, pp. 199-201, 1977.

[15] S. Hignett and L. McAtamney, "Rapid entire body assessment (reba)," Applied Ergonomics, vol. 31, no. 2, pp. 201-205, 2000.

[16] J. Diego-Mas and J. Alcaide-Marzal, "Using kinect sensor in observational methods for assessing postures at work," Applied Ergonomics, vol. 45, no. 4, pp. 976-985, 2013.

[17] A. Pandey and R. Alami, "Towards human-level semantics understanding of human-centered object manipulation tasks for hri: Reasoning about effect, ability, effort and perspective taking," International Journal of Social Robotics, vol. 6, no. 4, pp. 593-620, 2014.

[18] K. Ikuta, H. Ishii, and M. Nokata, "Safety evaluation method of design and control for human-care robots," The International Journal of Robotics Research, vol. 22, no. 5, pp. 281-297, 2003.

[19] E. A. Sisbot et al., "A human aware mobile robot motion planner," IEEE Transactions on Robotics, vol. 23, no. 5, pp. 874-883, 2007.

[20] E. A. Sisbot and R. Alami, "A human-aware manipulation planner," IEEE Transactions on Robotics, vol. 28, no. 5, pp. 1045-1057, 2012.

[21] F. Stulp, J. Grizou, B. Busch, and M. Lopes, "Facilitating intention prediction for humans by optimizing robot motions," in Proc. IEEE International Conference on Intelligent Robots and Systems (IROS'15'), Hamburg, Germany, 2015, pp. 1249-1255.

[22] H. B. Suay and E. A. Sisbot, "A position generation algorithm utilizing a biomechanical model for robot-human object handover," in Proc. IEEE International Conference on Robotics and Automation (ICRA'15), Seattle, Washington, USA, 2015, pp. 3776-3781.

[23] G. Li and P. Buckle, "Current techniques for assessing physical exposure to work-related musculoskeletal risks, with emphasis on posture-based methods," Ergonomics, vol. 42, no. 5, pp. 674-695, 1999.

[24] J. Nielsen, Usability engineering. Amsterdam, Netherlands: Elsevier, 1994.

[25] E. Hall, The Hidden Dimension. Garden City, NY, USA: Doubleday, 1966.

[26] E. T. Hall, "A system for the notation of proxemic behavior," American Anthropologist, vol. 65, no. 5, pp. 1003-1026, 1963.

[27] G. Maeda, M. Ewerton, D. Koert, and J. Peters, "Acquiring and generalizing the embodiment mapping from human observations to robot skills," IEEE Robotics and Automation Letters, vol. 1, no. 2, pp. 784-791, July 2016.

[28] E. Granjean, Fitting the Task to the Man: an Ergonomic Approach. London, England: Taylor Francis ltd, 1969.

[29] J. Brooke, "Sus-a quick and dirty usability scale," Usability Evaluation in Industry, vol. 189, no. 194, pp. 4-7, 1996. 